www.jmscr.igmpublication.org

Index Copernicus Value: 79.54

ISSN (e)-2347-176x ISSN (p) 2455-0450

crossref DOI: https://dx.doi.org/10.18535/jmscr/v7i4.12

\title{
Prediction of mortality risk by application of Pediatric Risk of Mortality (PRISM) score in tertiary care Pediatric Intensive Care Unit-A prospective observational study
}

\author{
Authors \\ Dr Pratik Kumar Dey ${ }^{1}$, Dr J Bikrant Kumar Prusty ${ }^{2}$, Dr Tushita Ghosh ${ }^{3}$, \\ Dr Satish Kumar ${ }^{4}$, Prof. Jasashree Choudhury ${ }^{5}$ \\ ${ }^{1,3,4}$ Post graduate student, ${ }^{2}$ Asst Prof., ${ }^{5}$ Professor \\ Department of Pediatrics, IMS \& SUM Hospital, Bhubaneswar, Odisha, India \\ Corresponding Author \\ Dr J Bikrant Kumar Prusty \\ Email: drjasashree@gmail.com, Mobile no-9437160366
}

\begin{abstract}
Aim: Our aim was to estimate the prediction of mortality by application of Pediatric Risk of Mortality (PRISM) score in a tertiary care pediatric intensive care unit (PICU) patients.

Methods: 225 children admitted to the PICU of a tertiary care hospital over a period of 24 months were enrolled in this cross-sectional observational study. PRISM III score was estimated using variables after admission in first 24 hours. The outcome was defined as survivors and deceased.

Results: Of 225 patients, 44 died and 181 survived. By PRISM score 49 children had the score of 1-9. 45 patients had PRISM score of $<15$, Maximum number of cases (113 patients) had PRISM score between 1630, 29 patients had a prism score ranging from 31-45 and 38 cases had a high PRISM score of $>45$. The recovery rate was $100 \%$ in the first group (scores <15), 99\% in the second group (score 16-30) while $75.86 \%$ recovery was seen in the 3rd group (score 31-45) and only 5.26\% in the fourth group (scores > 45). The proportion of deaths which was only 1\% among children with the PRISM scores of 16-30, showed a gradual increase with increase in score value, reaching $94.7 \%$ among the children with a PRISM scores of $>45$.

Conclusion: PRISM score has good predictive value in assessing the probability of mortality in relation to children admitted to a pediatric intensive care unit.
\end{abstract}

\section{Introduction}

Technological advances in Paediatric Intensive Care Unit (PICU) has resulted in a more sophisticated care for children and adolescents, making these units prepared to treat cases of high complexity at a high cost. However, the technology available has not been sufficient enough in improving the quality of patient care and higher ability to predict life expectancy becomes an issue that could increase the suffering and prolong death process ${ }^{1}$. The Global Scenario: Leading causes of severe illness in PICU is mainly due to Sepsis and ARDS, which are also significant causes of morbidity and mortality 
worldwide. The mortality rate of sepsis among children from PICU in developing countries is higher than $50 \%^{2}$. World Health Organisation (WHO) has shown that $80 \%$ of death in children < 4 years can be classified as sepsis-related deaths. WHO also estimated that every year 10 million children die of which $99 \%$ occurs in developing countries $^{3}$. As per the recent survey by WHO, Pneumonia is the major reason behind under 5 mortality in children ${ }^{[4]}$.

The intensive care for Paediatric and Neonatal diseases has improved over the recent times in India. With increase in demand for specialised intensive care unit services in rural India, many tertiary care hospitals have been established with excellent infrastructure and dedicated manpower. The outcome of intensive care has not been widely reported yet but the need for sophisticated equipment and aggressive treatment is still under developing stage. The practice of paediatric critical care is dynamic and evolving. Paediatric population is a vulnerable group necessitating standard care for medically ill children. However, standard care is not well-defined for paediatric critical care as most of the PICU protocols are actually extrapolation of adult critical care. The lack of consistency, reliability and accuracy in a physician's subjective opinions regarding patient's mortality demands the use of a standard objective and reproducible clinical prognostic scoring system $^{4}$. An ideal scoring system is one that is institution and population independent, wellcalibrated with a high level of discrimination, uses easily recordable variables and has the ability to predict the quality of life after critical care discharge. Previously, scoring systems were developed for trauma patients and were either specific anatomical methods (abbreviated injury scale-1974) or specific physiological methods (trauma index 1971, GCS in 1974 and sepsis score in 1983) $)^{5}$. The first scoring system in PICU was the Therapeutic Intervention Scoring System (TISS) in 1974 and it was mainly based on the fact that "therapeutic intensity defines the severity of illness" "5.Then came the APACHE (Acute
Physiology and Chronic Health Evaluation) score in 1981 which was based on 34 laboratory and clinical parameters. The first physiology based scoring system to assess the severity of acute illness in patients admitted to PICU was Physiology Stability Index (PSI) in 1984. But it was time consuming and dealt with 34 variables. Finally in 1996, PRISM Score was introduced. The score describes the illness according to the physiological derangements detected during clinical evaluation. It uses 14 parameters (physiological and lab data) and the severity values are assessed within the first 24 hours .Besides PRISM, another prognostic score being widely used is Paediatric Index of Mortality (PIM) score. This score is widely used in PICUs across New Zealand, Australia and United Kingdom .PRISM score, being a simple and effective tool for assessing the severity of illness in a child, this study was conducted with the aim and objective:

$>$ To find the performance of PRISM score in predicting mortality in patients admitted to our PICU.

$>$ To compare the predicted mortality with the observed mortality.

$>$ Secondary outcomes like the disease profile of patients admitted to PICU and the associated morbidity was studied.

\section{Methods}

This was a prospective observational study to evaluate the outcome of patients admitted to the paediatric intensive care unit (PICU) of IMS \& SUM hospital by using Paediatric Risk of Mortality Score (PRISM).

Place of study: This study was conducted in the Paediatric Intensive Care Unit of the Department of Paediatrics in the Institute of Medical Sciences and SUM Hospital (IMS \& SH) which a tertiary care centre and also one of the premier medical college and teaching hospital in Odisha. The PICU has a capacity of 5 beds and is well equipped with all the necessary equipments like monitors and mechanical ventilators. It is also backed by $24 \mathrm{hrs}$ in house laboratory facility, portable X-Ray, 
bedside dialysis, ECHO, Ultrasonography and ABG facilities.

Sample Size: As there were around 20-30 admissions on an average per month, the total admissions to the PICU was 278 during the study period .After excluding 53 cases, 225 cases were studied in detail. 30 cases were admitted on the basis of Post-operative stabilisation, 12 patients left against medical advice and 11 died within 8 hours of admission. All the consecutive patients admitted to the PICU fulfilling the inclusion criteria were the participants.

\section{Inclusion Criteria}

- All children between 1 month to14 years age group admitted directly to PICU, whose caretakers gave consent.

- Any patients admitted to paediatric ward and shifted within 24 hours to PICU were also studied.

- Surgical cases admitted to PICU for preoperative critical condition were also studied.

\section{Exclusion Criteria}

- Neonates are excluded from the study.

- Post-operative patients shifted from OT to PICU for stabilisation

- Patients staying for less than 8hours in PICU.

- Those patients who leave the hospital against medical advice are excluded as their outcome cannot be confirmed.

All the paediatric patients between 1 month to14years admitted to PICU, meeting the above criteria were included in the study. Following admission, a detailed history was taken, followed by thorough general and systemic examinations along with necessary investigations were done within 12 hours of admission, preferably within 4 hours. Basically considering the various variables in PRISM III score, all the necessary investigations required to determine the PRISM III score were within our PICU protocol ie.ABG analysis (for $\mathrm{pH}, \mathrm{PCo} 2, \mathrm{PaO} 2$, Total $\mathrm{Co} 2$, blood Glucose, serum Potassium), Complete Blood Count (for TWBC and TPC), PT/INR study, RFT (for BUN and Creatinine). ABG was done by drawing small amount of arterial blood and the analysis was obtained from a blood gas analyser. Total $\mathrm{CO} 2$ was calculated as $0.23 \times \mathrm{pCO} 2+$ Bicarbonate. Complete Blood count is being done by collecting around $2.5 \mathrm{ml}$ of blood in an EDTA vial and put on an automated haematology analyser (Sysmex 1000). The PRISM score is a measure of illness severity based on the abnormality observed in the bedside examination and laboratory assessment. Therefore all the patients were further evaluated by the following study variables which included 17 variables of PRISM III score namely (systolic blood pressure, temperature, mental status, heart rate, pupillary reflexes, $\mathrm{pH}, \mathrm{pCo} 2$, paO2, total $\mathrm{Co} 2$, potassium, BUN, Creatinine, Glucose, TWBC, TPC, PT/APTT) were assessed within 12 hours of PICU admission and the most abnormal parameter recorded within 12 hours of admission were considered. The PRISM score was calculated and the cases were followed till discharge or death.

Indications for PICU admission:

1) All patients requiring mechanical ventilation.

2) Patients with impending respiratory failure.

- Upper airway obstruction

- Lower airway obstruction

- Alveolar disease

- Unstable airway

3) All paediatric patients after successful resuscitation.

4) Comatose and disoriented patients

- Meningitis, Encephalitis

- Hepatic encephalopathy

- Cerebral/Complicated Malaria

- Head injury

- Poisoning/assault

- Status Epilepticus

5) All types of shock / hemodynamic instability

- Septic Shock

- Hypovolemic shock 
- Bleeding emergencies such as GI bleeding

- Bleeding diastheis, DIC

- Neurogenic Shock

- Cardiogenic Shock

- Multiple Trauma

6) Hypertensive Emergencies

7) Severe acid base disorders

8) Severe electrolyte abnormalities

9) Acute Renal Failure: Patients requiring dialysis.

10) Hematological cases mostly leukemias and lymphomas.

11) Acute Hepatic Failure.

Statistical analysis: All the 17 variables of the PRISM III were assessed and the most abnormal parameters were recorded within 12 hours of PICU admission were considered. The PRISM III score was calculated and the data was analysed using spss-22.The association between the study variables namely Age, Gender, Order of birth, Socio-economic status, Use of mechanical ventilation, Use of total parenteral nutrition, Use of ionotropic drugs, Duration of PICU stay,
Primary system affected and Outcome were tested using the Chi square test for categorical variables, as appropriate. Further the goodness of prediction was assessed by using area under Receiver Operating Characteristic (ROC) curve analysis. Bivariate logistic regression analysis was done using SPSS 22.

\section{Results}

Out of total 278 admissions to the PICU during the study period, 53 cases (30 admitted for PostOperative stabilisation, 12 participants left against medical advice (LAMA) and 11 died within 8 hours of admission) were excluded from the study. Finally 225 cases were participants of the study out of which, 181(80.4\%) patients recovered and $44(19.6 \%)$ died. The association between various study variables and the outcome was analysed and a $p$ value of $<0.05$ was considered as statistically significant, Majority of the study population were within the age group of 5 to 10 years. Males outnumbered the female participants in each sub group.(table 1)

Table 1: Association of Demographic Profile with Outcome.

\begin{tabular}{|c|c|c|c|c|c|}
\hline \multicolumn{2}{|c|}{ Demographic profile } & Total n(\%) number & Recovery n (\%) & Death n(\%) & P value \\
\hline \multirow{3}{*}{ Sex } & Males & $\begin{array}{c}147(65 \%) \\
1147(100 \%)\end{array}$ & $113(76.87 \%)$ & $34(23.12 \%)$ & 0.064 \\
& Females & $78(35 \%)$ & $68(87.17 \%)$ & $10(12.82 \%)$ & 8 \\
\cline { 2 - 6 } & $<12$ & 59 & 51 & 11 \\
\multirow{3}{*}{ Age } & $13-60$ months & 45 & 34 & 15 \\
\cline { 2 - 6 } & $61-120$ months & 85 & 70 & 10 \\
\end{tabular}

(Std.Error-3.407, df=3, $\mathrm{x}^{2}-3.776$ )

Male children were the majority 147 (65\%) with a survival rate of $76.87 \%$. whereas female patients were $78(35 \%)$ with a survival gender rate of $87.17 \%$. Though the survival rate was more in female participants there was no statistically significant association between the gender and the outcome ( $\mathrm{p}=0.064)$.

There were $59(26.2 \%)$ patients aged $<12$ months, $45(20 \%)$ between $13-60$ months and $85(37.7 \%)$ patients aged 61-120 months and 36(16\%) patients aged $>120$ months. The recovery rates in these four age groups were $85.9 \%, 78.7 \%$ and $83.95 \%$ and $70.37 \%$ respectively. The mean age for the study population was $68.37 \pm 6.8$ months. There was no statistically significant association of age and the outcome $(\mathrm{p}=0.153)$. 
Out of total 44 deaths, 29(32.2\%) belonged to the was statistically significant ( $p$ value-0.001). group with lower socio-economic status which

Table 2: Association of Prism Score with Mortality

\begin{tabular}{|c|c|c|c|c|}
\hline PRISM SCORE & $\begin{array}{c}\text { RECOVERY } \\
\text { n }(\%)\end{array}$ & $\begin{array}{c}\text { DEATH } \\
\mathrm{n}(\%)\end{array}$ & $\begin{array}{c}\text { TOTAL } \\
\mathrm{n}(\%)\end{array}$ & $\mathrm{P}$-value \\
\hline$<15$ & $45(100 \%)$ & 0 & $45(100 \%)$ & \multirow{4}{*}{0.001} \\
\hline $16-30$ & $112(99 \%)$ & $1(1 \%)$ & $113(100 \%)$ & \\
\hline $31-45$ & $22(75.86 \%)$ & $7 \quad(24.13 \%)$ & $29(100 \%)$ & \\
\hline$>45$ & $2(5.26 \%)$ & $36(94.73 \%)$ & $38(100 \%)$ & \\
\hline
\end{tabular}

The mean PRISM score of the study population was $27.83 \pm 2.14$ (95\% CI 25.69 - 29.97).The minimum PRISM Score of the study population was 5 and the maximum score was 66 . The mean PRISM score of the group which survived was $21.09 \pm 1.19(95 \%$ CI $19.9-22.28)$ and the group which died was $55.55 \pm 2.96$ (95\% CI $52.59-$ 58.51). On the basis of PRISM score all the subjects were divided into four categories . 45 patients had PRISM score of <15, Maximum number of cases (113 patients) had PRISM score between 16-30 , 29 patients had a prism score ranging from 31-45 and 38 cases had a high PRISM score of $>45$. (table 2 ) The recovery rate was $100 \%$ in the first group (scores <15), $99 \%$ in the second group (score 16-30) while $75.86 \%$ recovery was seen in the $3^{\text {rd }}$ group (score $31-45$ ) and only $5.26 \%$ in the fourth group (scores $>45$ ). The proportion of deaths which was only $1 \%$ among children with the PRISM scores of 16-30, showed a gradual increase with increase in score value, reaching $94.7 \%$ among the children with a PRISM scores of $>45$.The PRISM score had a statistically significant correlation with the outcome $\left(\mathrm{x}^{2}=78,62, \mathrm{df}=3, \mathrm{p}=0.001\right)$. The causes of illness were grouped as per the primary system involved. Respiratory system $(n=89)$ constituted the major cause of illness at the time admission, followed by CNS $(n=45)$, Infections $(\mathrm{n}=22)$, GI (4). The others included CVS $(\mathrm{n}=7)$, Haematological $(\mathrm{n}=20)$, Renal $(\mathrm{n}=11)$ and Others $(n=27)$. The survival rate of patients with major causes of illness like Respiratory disorders was $92.13 \%$, CNS $84.44 \%$, CVS was $100 \%$, and Infectious diseases were $90.90 \%$. Higher mortality was seen in diseases involving GI, Renal and Haematological system i.e. $75 \%, 72.7 \%$ and $60 \%$ respectively. The cause of illness showed significant correlation with the outcome $(\mathrm{p}=$ 0.001). (fig 1)

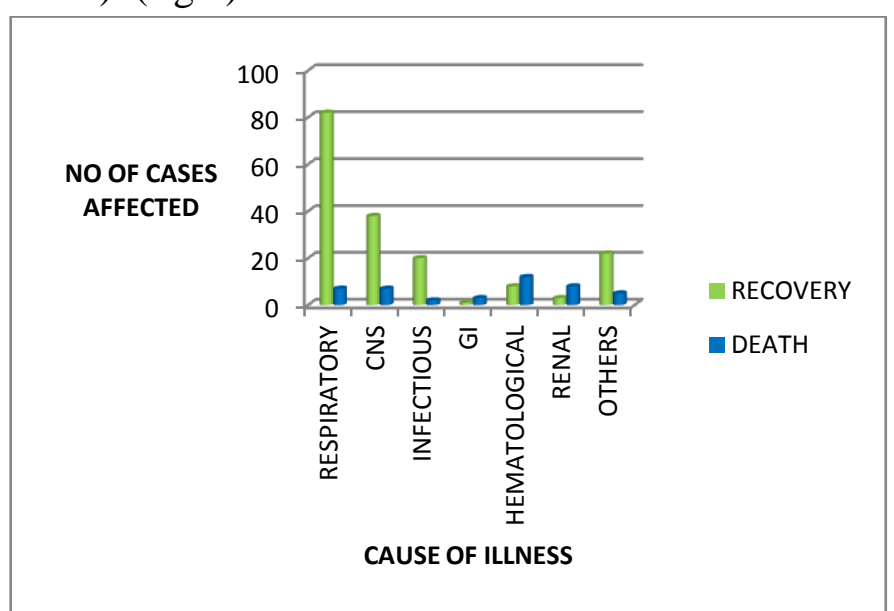

Fig-1: Association between systemic cause of illness \& outcome (Recovery/Death)

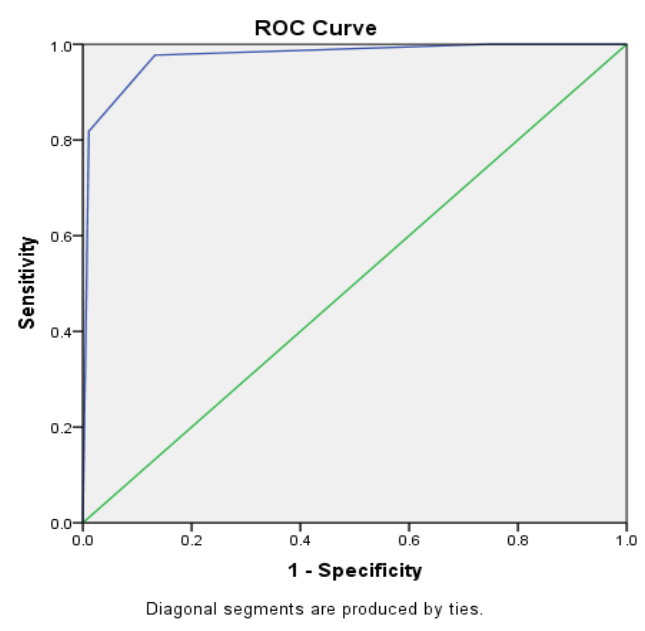

Fig-2: ROC curve 
Area under the curve is 0.974 and at a score of 50, PRISM score is $81.8 \%$ sensitive and $99 \%$ specific in predicting the mortality. The odds ratio of this logistic model was 1.29 with $95 \%$ confidence interval between 1.17 to 1.42 .For each unit change in PRISM score the odds of dying increases by $29 \%$.(fig 2)

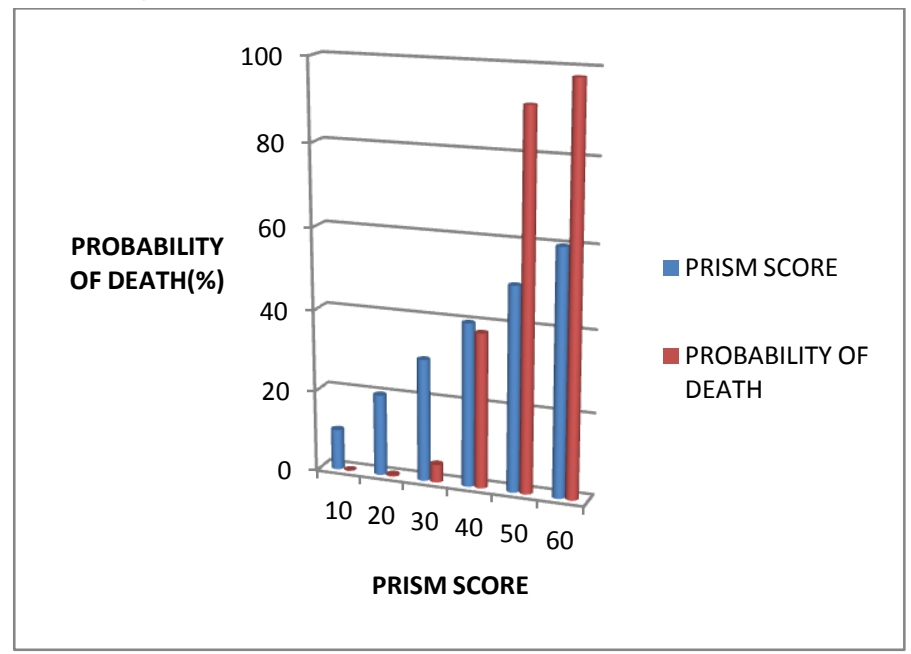

Figure 3: PRISM score in relation to Probability of Death

In the above Figure 9, the PRISM score and the probability of death is given for each score. The probability of death was $0.03 \%$ with a PRISM score of 10 and increased to $98 \%$ with a PRISM score of 60 .A score of 42 yielded $50 \%$ probability of death in PICU.

\section{Discussion}

In the present study done over a period of 1year, out of 225 participants $181(80.4 \%)$ cases recovered and got discharged and 44 (19.6\%) cases died, this is similar to other studies by Singhal .D et al ${ }^{6}$ and where the mortality was $18 \%$. However a study by Ahmed El Nawawy ${ }^{7}$ in Egypt showed a high death rate of $50.49 \%$.The outcome of an intensive care unit depends on the patient profile, the available services the lag period in admission to ICU and prompt initiation of treatment. As shown in the table no -3 the gender distribution showed 147(65.3\%) were males and 78 (34.6\%) were females. Males outnumbered females in each age category. Maximum number of patients belonged to category of 60 months to 120 months. The age of the patients was in the range of 1 month to 168 months. As shown in the table no - 3 mean age of the study population was 68.37 months $( \pm 6.80 \mathrm{~m}$ $95 \% \mathrm{CI})$. The mean age of patient who survived 66.20 months $( \pm 7.40 \mathrm{~m} \mathrm{95 \%} \mathrm{CI})$ and who died is 77.30 months $( \pm 16.20 \mathrm{~m} \mathrm{95 \%} \mathrm{CI).} \mathrm{The} \mathrm{age} \mathrm{and}$ gender distribution of the study population has no significant association with the outcome. This is in agreement with other Indian study by Mehta J S et $\mathrm{al}^{8}$, and Singhal D et al. ${ }^{7}$ The mean age of the study population is different from that of the original study by Pollack et $\mathrm{al}^{10}$ where they had a mean age of 33 months. As shown in the table no 4 patients belonging to lower socio-economic status had a significantly higher mortality rate with $\mathrm{p}$ value $<0.05$ which can be explained in terms of poor nutritional condition and delay in initiation of medical help .As evident in table no 5 the mean PRISM score of the study population was $27.83 \pm 2.14$ (95\% CI 25.69 - 29.97). Which is similar other studies ${ }^{9}$. The mean score of other studies was lesser than our study, 14.22 was the mean score in the study by Bilan et $\mathrm{al}^{11}$ The mean PRISM score of the group which survived was $21.09 \pm 1.19(95 \%$ CI $19.9-22.28)$ which is much smaller than the group which died with a score of $55.55 \pm 2.96$ ( 95\% CI $52.59-58.51$ ) which is statistically significant. This is higher than mean score of other studies which reflects the more serious clinical condition of patients at the time of presentation to the hospital. This may be due to delayed referral to a tertiary care centre in our set up. There was no death in the group with prism score less than 15. proportion of deaths which was only $1 \%$ among children with the PRISM scores of 16-30, showed a gradual increase with increase in score value, reaching $94.7 \%$ among the children with a PRISM scores of > 45. The PRISM score had a statistically significant positive correlation with the outcome $\left(x^{2}=78,62, d f=3, p=0.000\right)$. This is similar to other studies. ${ }^{6,7}$ As shown in table no- 6 the source of admission had no significant association with the outcome of the patients $(\mathrm{P}=0.096)$. However other studies suggested higher mortality in cases 
referred from other hospitals. As depicted in table no-7, majority of patients were admitted for respiratory illness number -89 (39.5\%) followed by Central nervous system diseases number 45 $(20 \%)$ and infectious $22(9.7 \%)$ cases like septicemia. The cause of illness has a significant association with mortality $(\mathrm{P}=0.001)$ This is similar in observation of another study ${ }^{7}$. Though maximum cases were with respiratory illness but there was good recovery rate in them (92.13\%). Patients admitted for Gastro intestinal illness had maximum mortality 3 out of 4 cases died. This may be explained as out of 4 cases with Gastro intestinal diseases, 3 cases were hepatic failure with encephalopathy .Patients with hematological conditions also had high mortality rate of $(60 \%)$.

As shown in table 8 , mechanical ventilation has a statistically significant correlation with mortality. As observed by $50 \%$ mortality in the group which needed mechanical ventilation $(\mathrm{P}=<0.001)$, which is in agreement with the study by Mukhtar et $\mathrm{al}^{12}$. This can be explained in terms of complications and nosocomial infections in patients requiring mechanical ventilation. As described in table no 10 the need of ionotropic support also had significant association with mortality $(\mathrm{P}=0.001)$, this is suggestive of more hemodynamic instability which had significantly higher mortality rate. This is similar to other study by Mukhtar et $\mathrm{al}^{12}$. As depicted in the table no 12 the duration of PICU stay also had a significant association with mortality, $(60.4 \%)$ mortality with PICU stay of more than 10 days. This is in agreement with study by Graziela et $\mathrm{al}^{13}$. However the original study by Pollack et al ${ }^{10}$ didn't show any association of length of stay with mortality. This may be explained in terms of the institutional policy regarding transfer of patients to high dependency units once they are stabilized in ICU. In the figure no - the ROC curve analysis shows an Area under the curve is 0.974 which is highly significant and this is in agreement with another Indian study by Madaan et $\mathrm{al}^{14}$ where their area under curve was 0.934 and Pollack et al, where the ROC curve had an area of $0.947 \pm 0.007$
.Though other studies had Area under curve was varied from $0.720^{7}$ to $0.820^{16}$. At a score of 50, PRISM score is $81.8 \%$ sensitive and $100 \%$ specific in predicting the mortality, which is different from a study by Bellard et $\mathrm{al}^{15}$ where they found an overall mortality of $16.7 \%$ with $89.2 \%$ accuracy at cut off score of 15.Another study from India by Thukral et $\mathrm{al}^{16}$ reported that PRISM score under predicted deaths in PICUS in Indian settings. In our study higher PRISM score predicted mortality with high sensitivity and specificity. As suggested in the table no 13, log odds of a child dying increases with 0.259 unit increase in the PRISM score. The odds ratio of this logistic model was 1.29 with $95 \%$ confidence interval between 1.17 to 1.42 .For each unit change in PRISM score the odds of dying increases by $29 \%$ which is different from the study by Singhal et $\mathrm{al}^{7}$ where the odds of death increased by only $12 \%$ this may explained in terms of the variation in the quality of care and outcome in different critical care set up.

\section{Conclusion}

The present study was done in a tertiary care referral hospital to evaluate the outcome of patients admitted to PICU by using PRISM score. The common illnesses for admission to PICU were respiratory cause. The mean PRISM score of patients who survived was higher as compared to those who succumbed. The duration of hospital stay was longer in patients who died than those who survived. The logistic regression analysis of PRISM score showed that it is a highly sensitive tool to predict the outcome. This study suggests that PRISM score at presentation may be used as a tool in predicting mortality in critically ill children. The probability of death increases significantly with increase in PRISM score. This score can be used as a tool to prioritize patients in a PICU in a resource limited setting like India and early referral of cases to tertiary care hospitals is needed for better survival of pediatric patients. 


\section{References}

1. Sarathi M, Lodha R, Vivekanandhan S, Arora NK. Adrenal status in children with septic shock using low-dose stimulation test. Peditr Crit Care. 2007; 8:23-8.

2. Bryce J, Boschi-Pinto C, Shibuya K, Black RE.WHO child health epidemiology reference group.WhO estimates of the causes of death in children. Lancet 2005; 365:1147-52.

3. Global Health Observatory (GHO) data.www.who.int

4. Gomke RJBJ, Bonsel GJ, Johannes Van Vught A. Effectiveness and efficiency of a Dutch Pediatric intensiveCare unit: Validity and application of the Pediatric Risk of mortality score. Critical Care Med 1994; 2:1477-1484.

5. Bhadoria Poonam, Bhagwat Amit. Severity Scoring Systems in Paediatric Intensive Care Units. Indian Journal of Anaesthesia 2008;52:Suppl(5):663-675

6. Singhal D, N. Kumar, J. M. Puliyel, S. K. Singh, V. Srinivas. Prediction of mortality by application of PRISM score in Intensive care unit. Indian Paediatrics 2001Jul;38(7):714-9.

7. Ahmed El-Nawawy. Evaluation of the outcome of patients admitted to PICU in Alexandria using the PRISM score. Journal of tropical paediatrics 2003 Apr;49(2):109-14..

8. Mehta $\mathbf{J}$ et al. PRISM III score as predictor of mortality in a paediatric intensive care unit of a tertiary care hospital.Journal of Paediatric Critical care. 2016 ;3 (4): 16-19.

9. Anthony RB, David H, Stephanie B, Sam J, Kathy R, Gale P, et al. assessment and optimization of mortality prediction tools for admissions to pediatric intensive care in the united kingdom. pediatrics. 2006; 117(e733).

10. Pollack MM, Kantilal M Patel, Urs E Ruttiman. PRISM III: An updated pediatric risk of mortality Score. CritCare Med 1996; 24: 743-752.

11. Bilan N. Risk of mortality in PICU, assessed by PRISM score. Pakistan Journal of Biological Sciences2009;12(6): 480-487.

12. Mukthar FR.A study on the prediction of illness related mortality of critically ill children by applying paediatric risk of mortality III score in paediatric medical intensive care unit patient. Sri Lanka Journal of Child Health, 2018; 47(2):118124.

13. Graziela DAC, Artur FD, Alexandre F, Thelma S.Application of the Pediatric Risk of Mortality Score (PRISM) score and determination of mortality risk factors in a tertiary pediatric intensive care unit. CLINICS. 2010; 65(11): 1087-1092.

14. Madaan G, Bhardwaj AK, Sharma PD, Dhanjal GS. Validity of PRISM score in prediction of mortality in North Indian pediatric intensive care unit. Indian J Child Health. 2014;1(3):105-8.

15. Bellad R. Outcome of Intensive Care Unit Patients Using Pediatric Risk of Mortality (PRISM) Score: Indian pediatrics. 2009 Dec;46(12):1091-2

16. Thukral A. Performance of Paediatric Risk of Mortality (PRISM), Paediatric Index of Mortality (PIM) and PIM2 in a paediatric intensive care unit in a developing country. Pediatr Crit Care Med 2006 Jul;7(4):35661. 